\title{
Can PET/CT Guide the Personalized Treatment of Patients with Gastroenteropancreatic Neuroendocrine Neoplasms?
}

G astroenteropancreatic neuroendocrine neoplasms (GEPs) arise from the diffuse neuroendocrine system. Their incidence has markedly increased over the past 3 decades, probably as a result of the improvement in imaging and biochemical methods of detection (1). They are a heterogeneous group of neoplasms with varying clinical expressions, presenting as either functioning or nonfunctioning, with an indolent clinical course in most patients. Functioning tumors are commonly associated with a typical hormonal syndrome directly related to a hormone secreted by the neoplasm; nevertheless, patients may have symptoms for many years before the correct diagnosis is made, because symptoms are often nonspecific and can be due to hormonal excess, local tumor growth, or metastatic spread (2).

Tumor grading and staging represent the main prognostic factors of these neoplasms. Apart from location, GEPs are graded according to proliferation activity, usually evaluated by the Ki67 index on tumor cells, which can have a strong impact on prognosis and therapy (3). The well-differentiated neoplasms, regardless of their benign or malignant behavior, are named neuroendocrine

\section{See page 1811}

tumors (NETs) and graded G1 (Ki67 < 2\%) or G2 (Ki67 of $2 \%-$ $20 \%)$; the poorly differentiated neoplasms are named neuroendocrine carcinomas and graded G3 (Ki67 > 20\%) (4). Both NETs and neuroendocrine carcinomas are also further classified according to the TNM staging system of the European Neuroendocrine Tumor Society and the American Joint Committee on Cancer/Union for International Cancer Control, to stratify them regarding prognosis (5). The recent advances in molecular biology have led to an increase in treatment options for these patients, but their management has become more complex, requiring a multidisciplinary approach.

Nuclear medicine has for several years played an important clinical role in imaging and treating GEPs. A well-known characteristic of most GEPs is overexpression of somatostatin receptors, which can successfully be visualized in vivo by somatostatin receptor imaging, using radiopharmaceuticals emitting single photons or positrons. Moreover, somatostatin analogs labeled with $\beta$-emitting radionuclides are target therapy for inoperable or metastasized GEPs (6).

In the current decade, PET/CT using ${ }^{68} \mathrm{Ga}$-DOTA-labeled somatostatin analogs has been introduced for the diagnostic work-

Received Sep. 26, 2014; revision accepted Sep. 30, 2014.

Published online Oct. 23, 2014.

COPYRIGHT (C) 2014 by the Society of Nuclear Medicine and Molecular Imaging, Inc.

DOI: $10.2967 /$ jnumed.114.147538 up of GEPs. In this issue of The Journal of Nuclear Medicine, Has Simsek et al. (7) propose the complementary use of ${ }^{68} \mathrm{Ga}-$ DOTATATE and ${ }^{18}$ F-FDG PET/CT in patients with GEPs for assessing the relationship between in vivo uptake of the two radiopharmaceuticals and Ki67 indices in the management of therapy. Previous studies had reported that high ${ }^{18} \mathrm{~F}-\mathrm{FDG}$ uptake is usually associated with more aggressive GEPs and a less benign prognosis, whereas higher uptake of ${ }^{68} \mathrm{Ga}$-DOTATATE is characteristic of lowgrade neoplasms versus high-grade ones $(8,9)$.

In the series of Has Simsek et al. (7), 27 patients were prospectively evaluated and classified as having NETs (10 G1 and 15 G2) or neuroendocrine carcinoma ( 2 G3). The patients with G2 NETs were then divided into two groups: $2 \mathrm{a}$ (Ki67 of 3\%-9\%) and 2b (Ki67 of $10 \%-20 \%$ ). According to the PET/CT findings, GEPs were further dichotomized as showing either predominantly ${ }^{68} \mathrm{Ga}$-DOTATATE uptake or predominantly ${ }^{18} \mathrm{~F}$-FDG uptake, considering both the number of detected lesions and the maximum standardized uptake value of the radiopharmaceutical. On a lesion basis, the overall sensitivity of ${ }^{68} \mathrm{Ga}$-DOTATATE and ${ }^{18} \mathrm{~F}$-FDG PET/CT was $95 \%$ and $37 \%$, respectively. Concordant findings were considered to have predominantly ${ }^{68} \mathrm{Ga}$-DOTATATE uptake if the Ki67 index was lower and predominantly ${ }^{18} \mathrm{~F}$-FDG uptake if the Ki67 index was higher. Taking into account a cutoff value of $9 \%$ for Ki67, 19 of the 27 patients showed concordant findings and 8 showed discordant ones. ${ }^{68} \mathrm{Ga}$-DOTATATE uptake was predominant in 5 patients with a Ki67 index of more than $9 \%$, and ${ }^{18} \mathrm{~F}$-FDG uptake was predominant in 3 patients with a $\mathrm{Ki} 67$ index of $9 \%$ or less; these last 3 patients had G2a disease. The combined PET/CT assessment led to a change in treatment options in 59\% of patients (16/27).

Kayani et al. (8), in studying a group of 38 patients with neuroendocrine neoplasms, including $28 \mathrm{GEPs}$, found that 3 patients lacked uptake of both ${ }^{68} \mathrm{Ga}$-DOTATATE and ${ }^{18} \mathrm{~F}-\mathrm{FDG}$ (1 G1, 1 $\mathrm{G} 2$, and $1 \mathrm{G} 3$ ). In the remaining 35 patients, predominantly ${ }^{68} \mathrm{Ga}-$ DOTATATE uptake was observed in all $21 \mathrm{G} 1$ neoplasms and predominantly ${ }^{18}$ F-FDG uptake in all $6 \mathrm{G} 3$ neoplasms; of the $6 \mathrm{G} 2$ neoplasms, 3 had predominantly ${ }^{68} \mathrm{Ga}$-DOTATATE uptake and the other 3 predominantly ${ }^{18} \mathrm{~F}-\mathrm{FDG}$ uptake. The combined use of the two radiopharmaceuticals caused the choice of therapy to be changed from radionuclide to systemic chemotherapy in $25 \%$ of the patients with intermediate- or high-grade neoplasms (4/16: $1 \mathrm{G} 2$ and $3 \mathrm{G} 3$ ). The conclusions of this retrospective study were that tumor grade influenced tracer avidity but that it was difficult to establish a full link between radiopharmaceutical uptake and histopathologic indices of tumor proliferation, mainly because of the large number of lesions in several patients, but also taking into account that percutaneous biopsy may not reliably reflect in vivo tumor heterogeneity.

The clinically useful complementary role of ${ }^{18}$ F-FDG to somatostatin receptor imaging has been subsequently confirmed in a large prospective study enrolling 96 patients with neuroendocrine neoplasms (82 GEPs, 7 in the lungs and 7 with liver metastases of unknown primary) (10). The sensitivity of ${ }^{18} \mathrm{~F}$-FDG PET was significantly higher for neoplasms with a Ki67 index of $2 \%$ or more $(80 \%$ 
vs. $41 \%$ ), in particular for pancreaticoduodenal and poorly differentiated neuroendocrine carcinomas. However, the sensitivity of ${ }^{111}$ Inpentetreotide scintigraphy was the same (i.e., 87\%) for all neoplasms regardless of Ki67 index. Moreover, with a cutoff value of $15 \%$ for the proliferation index, ${ }^{18}$ F-FDG PET sensitivity was $92 \%$ and $53 \%$ for neoplasms above and below this value, respectively, whereas ${ }^{111}$ In-pentetreotide sensitivity was $90 \%$ and $69 \%$, respectively.

More recently, Naswa et al. (11) retrospectively compared the diagnostic performance of ${ }^{68} \mathrm{Ga}$-DOTANOC with that of ${ }^{18} \mathrm{~F}-\mathrm{FDG}$ PET/CT in 51 patients with GEPs. Ki67 values were available for only 25 patients; therefore, no correlation was made between tracer avidity and proliferation index. Nevertheless, on visual analysis, the degree of ${ }^{68} \mathrm{Ga}$-DOTANOC uptake in well-differentiated GEPs seemed higher than the degree of ${ }^{18} \mathrm{~F}$-FDG uptake and vice versa. The addition of ${ }^{18} \mathrm{~F}$-FDG imaging to ${ }^{68} \mathrm{Ga}$-DOTANOC changed the treatment of 3 patients.

The possible prognostic value of ${ }^{18} \mathrm{~F}-\mathrm{FDG}$ PET in GEPs has been evaluated in two prospective studies. In the first (12), 38 patients with histologically proven well-differentiated metastatic NETs of the digestive tract or upper airways were enrolled. At the 6-mo follow-up, 16 patients showed early progressive disease and 22 stable disease; the median follow-up of surviving patients $(n=$ 27) was 20 mo. At visual analysis, ${ }^{18}$ F-FDG PET was positive for uptake in 15 patients and was strongly related to the Ki67 findings. Fourteen of the 15 positive patients had early progressive disease, and 21 of the 23 negative ones had stable disease. Moreover, survival was better among ${ }^{18}$ F-FDG-negative patients than among positive ones for both overall survival and progression-free survival; at multivariate analysis, the PET findings were independently predictive of progression-free survival.

In the second study (9), 98 patients with neuroendocrine neoplasms (83 GEPs, 8 bronchopulmonary neoplasms, and 7 liver metastases with an unknown primary) were enrolled. The prognostic value of ${ }^{18} \mathrm{~F}-\mathrm{FDG}$ uptake (evaluated by maximum standardized uptake value), Ki67, chromogranin A, and liver metastases was assessed. PET sensitivity was $58 \%$ on a patient basis; ${ }^{18} \mathrm{~F}-\mathrm{FDG}$ results were positive in $40 \%$ of patients with a Ki67 index of less than $2 \%$, in $70 \%$ of patients with a Ki67 index of $2 \%-15 \%$, and in $93 \%$ of patients with a Ki67 index of more than $15 \%$. The mean follow-up time after ${ }^{18} \mathrm{~F}-\mathrm{FDG}$ imaging was $11.5 \mathrm{mo}$. Thirteen of the $57{ }^{18} \mathrm{~F}-\mathrm{FDG}$-positive patients died, compared with 1 of $41(2 \%){ }^{18}$ F-FDG-negative patients. Patients in the PETpositive group had significantly lower overall and progression-free survival than the PET-negative group. Finally, in a multivariate analysis, a maximum standardized uptake value of more than 3 was the only predictor of progression-free survival.

The study of Has Simsek et al. (7) has clearly indicated that the combination of ${ }^{68} \mathrm{Ga}$-DOTATATE and ${ }^{18} \mathrm{~F}$-FDG imaging can be clinically useful for determining the optimal choice of personalized treatment for GEP patients. Their findings suggest that this approach is especially of value in G2 GEPs, considering that G2b is a higher-grade tumor, thus helping the clinicians overcome the limitations of histopathologic grading. Indeed, biopsy often may not completely reflect in vivo tumor heterogeneity, and the optimal method to determine the Ki67 index is still debated (13). The shortcomings of Ki67 are accentuated in NETs, as differences in the range of $1 \%$ to $5 \%$ can alter tumor grade, with potential implications for treatment (14). If tumor cell proliferative rate is an important factor in determining prognosis, and immunohistochemical analysis with Ki67 is becoming more widely used to quantify it, how to perform this count and how to improve the diagnostic and prognostic value of this proliferation marker has yet to be fully defined. Moreover, more studies are needed to better establish the cutoffs for the clinically relevant categories of GEPs.

Surgical resection is often the best option for most localized GEPs, but the treatment of inoperable cases and treatment after surgery have evolved, with various therapies now being available (15). The data of Has Simsek et al. (7) should be confirmed in a larger series but clearly demonstrate the possible central role of PET/CT in guiding the management of patients with GEPs, especially if combined with clinicopathologic data for optimizing individual treatment.

\section{DISCLOSURE}

No potential conflict of interest relevant to this article was reported.

Orazio Schillaci
University of Rome Tor Vergata
Viale G. Mazzini 121
Rome 00195, Italy
E-mail: orazio.schillaci@uniroma2.it

\section{REFERENCES}

1. Yao JC, Hassan M, Phan A, et al. One hundred years after "carcinoid": epidemiology of and prognostic factors for neuroendocrine tumors in 35,825 cases in the United States. J Clin Oncol. 2008;26:3063-3072.

2. Oberg K. State of the art and future prospects in the management of neuroendocrine tumors. Q J Nucl Med. 2000;44:3-12.

3. Klimstra DS, Modlin IR, Coppola D, Lloyd RV, Suster S. The pathologic classification of neuroendocrine tumors: a review of nomenclature, grading, and staging systems. Pancreas. 2010;39:707-712.

4. Rindi G, Arnold R, Bosman FT, et al. Nomenclature and classification of neuroendocrine neoplasms of the digestive system. In: Bosman FT, Carneiro F, Hruban RH, Theise ND, eds. WHO Classification of Tumors of the Digestive System. 4th ed. Lyon, France: IARC Press; 2010:13-14.

5. Klöppel G. Classification and pathology of gastroenteropancreatic neuroendocrine neoplasms. Endocr Relat Cancer. 2011;18(suppl 1):S1-S16.

6. Volterrani D, Orsini F, Chiacchio S, Bodei L. Multiagent targeting of neuroendocrine neoplasms. Clin Transl Imaging. 2013;1:407-421.

7. Has Simsek D, Kuyumcu S, Turkmen C, et al. Can complementary ${ }^{68} \mathrm{Ga}-$ DOTATATE and ${ }^{18} \mathrm{~F}$-FDG PET/CT establish the missing link between histopathology and therapeutic approach in gastroenteropancreatic neuroendocrine tumors? J Nucl Med. 2013;55:1811-1817.

8. Kayani I, Bomanji JB, Groves A, et al. Functional imaging of neuroendocrine tumors with combined PET/CT using ${ }^{68} \mathrm{Ga}$-DOTATATE (DOTA-DPhe1,Tyr3octreotate) and ${ }^{18}$ F-FDG. Cancer. 2008;112:2447-2455.

9. Binderup T, Knigge U, Loft A, Federspiel B, Kjaer A. ${ }^{18}$ F-fluorodeoxyglucose positron emission tomography predicts survival of patients with neuroendocrine tumors. Clin Cancer Res. 2010;16:978-985.

10. Binderup T, Knigge U, Loft A, et al. Functional imaging of neuroendocrine tumors: a head-to-head comparison of somatostatin receptor scintigraphy, ${ }^{123} \mathrm{I}-$ MIBG scintigraphy, and ${ }^{18}$ F-FDG PET. $J$ Nucl Med. 2010;51:704-712.

11. Naswa N, Sharma P, Gupta SK, et al. Dual tracer functional imaging of gastroenteropancreatic neuroendocrine tumors using ${ }^{68} \mathrm{Ga}-\mathrm{DOTA}-\mathrm{NOC}$ PETCT and ${ }^{18}$ F-FDG PET-CT: competitive or complimentary? Clin Nucl Med. 2014;39:e27-e34.

12. Garin E, Le Jeune F, Devillers A, et al. Predictive value of ${ }^{18} \mathrm{~F}$-FDG PET and somatostatin receptor scintigraphy in patients with metastatic endocrine tumors. J Nucl Med. 2009;50:858-864.

13. Klimstra DS, Modlin IR, Adsay NV, et al. Pathology reporting of neuroendocrine tumors: application of the Delphic consensus process to the development of a minimum pathology data set. Am J Surg Pathol. 2010;34:300-313.

14. Tang LH, Gonen M, Hedvat C, Modlin IM, Klimstra DS. Objective quantification of the Ki67 proliferative index in neuroendocrine tumors of the gastroenteropancreatic system: a comparison of digital image analysis with manual methods. Am J Surg Pathol. 2012;36:1761-1770.

15. Kim KW, Krajewski KM, Nishino M, et al. Update on the management of gastroenteropancreatic neuroendocrine tumors with emphasis on the role of imaging. AJR. 2013;201:811-824. 